\title{
On the Growth of Functions of Mean Type
}

\author{
By W. H. J. Fuchs \\ (Received 14th February, 1950. Read 5th May, 1950. \\ Revised 21st July, 1953.)
}

1. V. Bernstein, N. Levinson, R. P. Boas, Jr., and others have investigated under what conditions on the sequence $\left\{\lambda_{n}\right\}$

$$
\varlimsup_{n \rightarrow \infty} \lambda_{n}^{-1} \log \left|f\left(\lambda_{n}\right)\right|=\varlimsup_{x \rightarrow \infty} x^{-1} \log |f(x)|
$$

for all functions $f(z)$ regular and of suitably restricted growth in the halfplane $x \geqslant 0$. (For references see [1].)

In this note the restrictions on $f(z)$ are that $f(z)$ is regular. and not identically zero in $|\arg z|<\frac{1}{2} \pi$, continuous in $|\arg z| \leqslant \frac{1}{2} \pi$, and that for some positive $B$

$$
|f(z)|<e^{B|z|}, \quad|f(i y)|<e^{\pi L|y|} \quad(x \geqslant 0)
$$

where $L>0$. Of the $\lambda_{n} I$ shall assume that they are real positive numbers and that

$$
\lambda_{n+1}-\lambda_{n} \geqslant c>0 \quad(n=1,2, \ldots)
$$

The method of this paper could be adapted to deal with the more general $\left\{\lambda_{n}\right\}$ considered by Levinson. I shall write

$$
\Lambda(r)=L r+l(r)
$$

for the number of $\lambda_{n}$ not exceeding $r$.

N. Levinson proved [4, p. 108, Th. 38; $t(u)=|l(u)|-l(u)]$ :

Theorem A. If $f(z)$ and $\left\{\lambda_{n}\right\}$ are subject to the conditions stated and if

$$
\begin{aligned}
& \lim _{r \rightarrow \infty} l(r) / r \text { exists, } \\
& \int_{1}^{\infty} u^{-2} l(u) d u=\infty
\end{aligned}
$$

and

$$
\int_{1}^{\infty} \frac{|l(u)|-l(u)}{u^{2}} d u<\infty
$$

then (1.1) holds. 
The condition (1.5) implies that the limit in (1.4) is not negative. If this limit is positive, then (1.4) implies (1.5) and (1.6). The most interesting case is

$$
l(r) / r \rightarrow 0 .
$$

I want to point out that a refinement of Theorem $A$ leads to a best possible result. This is most easily stated in terms of the function

$$
\begin{aligned}
\phi(r) & =\int_{1}^{r} u^{-1} d l(u)=\frac{l(r)}{r}+\int_{1}^{r} \frac{l(u)}{u^{2}} d u+\text { constant } \\
& =\sum_{\lambda_{n} \leqslant r} \lambda_{n}^{-1}-L \log r+\text { constant. }
\end{aligned}
$$

TheOREM B. Under the assumptions (1.2) and (1.3) the relation (1.1) holds if and only if

$$
\lim _{r \rightarrow \infty} \phi(r)=\infty
$$

$$
\text { for given } \epsilon>0, \phi(Y)-\phi(X)>-\epsilon \text { for } Y>X>K(\epsilon) \text {. }
$$

Because of (1.3) the conditions $(1.5)$ and $(1.8)$ are equivalent and it is easy to see that (1.4) and (1.6) imply (1.9), but the converse is not the case. Theorem B contains no condition like (1.4), but I shall show (§2) that under the hypotheses of Theorem $\mathrm{B}\left\{\lambda_{n}\right\}$ can be replaced by a sub-sequence for which (1.8), (1.9) and (1.7) hold. In proving the sufficiency part of Theorem B we may therefore assume (1.7), since (1.1) is certainly true for $\left\{\lambda_{n}\right\}$ if it is true for a sub-sequence of $\left\{\lambda_{n}\right\}$.

The proof that (1.7), (1.8) and (1.9) imply (1.1) can be carried out by the method of R. P. Boas [1] and I therefore omit it.

The necessity of the conditions (1.8) and (1.9) is proved by the construction of counter-examples in $\S \S 3-5$.

2. I prove first that under the hypotheses (1.8) and (1.9)

$$
\underline{\lim } l(r) / r \geqslant 0 \text {. }
$$

(1.8) implies that $\overline{\lim } l(r) / r \geqslant 0$. If $\underline{\lim } l(r) / r=-\alpha<0$, then there is a sequence of $Y \rightarrow \infty$ such that $l(Y) / Y<-\alpha+\frac{1}{2} \epsilon$. For every such $Y$ define $X$ as the largest $r<Y$ such that $l(r) / r>-\frac{1}{2} \epsilon$. Since $\varlimsup \lim l(r) / r \geqslant 0$, such an $X$ exists for all large $Y$ and $X \rightarrow \infty$ with $Y$. Then

$$
\phi(Y)-\phi(X)=\frac{l(Y)}{\bar{Y}}-\frac{l(X)}{\bar{X}}+\int_{\boldsymbol{X}}^{\boldsymbol{Y}} \frac{l(u)}{u^{2}} d u \leqslant-\alpha+\epsilon,
$$

which contradicts (1.9). 
I show next that it is possible to replace $\left\{\lambda_{n}\right\}$ by a sub-sequence satisfying (1.8), (1.9) and

$$
\overline{\lim } l(r) / r=0 \text {. }
$$

This combined with (2.1) will prove that (1.7) holds for the new sequence.

I define

$$
-\epsilon(X)=\inf _{X<u<v}\{\phi(v)-\phi(u)\}
$$

The function $\epsilon(X)$ is a decreasing, positive function tending ${ }^{1}$ to 0 , by (1.9). Let $\left\{X_{n}\right\}$ be a sequence of positive numbers satisfying

$$
\begin{gathered}
X_{n+1}-X_{n} \rightarrow \infty \\
X_{n+1} / X_{n} \rightarrow 1 \\
\left(X_{n+1}-X_{n}\right) / X_{n} \in\left(X_{n}\right) \rightarrow \infty .
\end{gathered}
$$

It is easily seen from (1.3) and the definitions of $l(r)$ and $\phi(r)$ that $l(r) / r$ and $\phi(r)$ vary by $o(1)$ only as $r$ varies in the interval $\left\langle X_{n}, X_{n+1}\right\rangle$. It will therefore be sufficient to verify (1.8), (1.9) and (2.2) for values of $r, X$ and $Y$ tending to infinity through the sequence $\left\{X_{n}\right\}$. There is nothing to prove if (2.2) holds to start with.

If $0<\beta<\overline{\lim } l(r) / r$, then

$$
\Sigma^{\prime} \frac{X_{\nu+1}-X_{v}}{X_{\nu+1}}=\infty,
$$

where the summation is over all those intervals $\left(X_{v}, X_{v+1}\right)$ containing at least $(L+\beta)\left(X_{v+1}-X_{v}\right)$ zeros. For suppose (2.3) false. Then, for a given $\delta>0$, there is a $T$ such that

$$
\sum_{X_{\nu}>\boldsymbol{T}}^{\prime} \frac{X_{v+1}-X_{v}}{X_{v+1}}<\delta .
$$

Now we estimate $\Lambda\left(X_{n}\right)$ by estimating separately the number of $\lambda$ 's $\leqslant T$, the number of $\lambda$ 's in intervals $\left(X_{v}, X_{v+1}\right)$ not contributing to $(2.3)$ and in intervals contributing to (2.3). This yields, using (1.3),

$$
\begin{aligned}
\Lambda\left(X_{n}\right) & \leqslant \frac{1}{c} T+(L+\beta) X_{n}+\frac{1}{c} \sum_{\substack{\Sigma_{i=\pi}^{\prime} \\
X_{v}>r}}\left(X_{v+1}-X_{v}\right) \\
& \leqslant \frac{1}{c} T+\left\{L+\beta+\frac{1}{c} \sum_{X_{v}>T} \frac{X_{v+1}-X_{v}}{X_{v+1}}\right\} X_{n} .
\end{aligned}
$$

\footnotetext{
${ }^{1}$ Thimite in this seotion are always taken as the variable tends to $\infty$.
} 
By (2.4) and the definition of $l(r)$ this would imply

$$
\varlimsup i \lim l(r) / r=\overline{\lim } l\left(X_{n}\right) / X_{n} \leqslant \beta,
$$

contrary to hypothesis.

We now choose a sequence $\left\{\eta_{n}\right\}$ of positive numbers tending to zero such that

$$
\Sigma^{\prime} \eta_{v} \frac{X_{v+1}-X_{v}}{X_{v+1}}=\infty
$$

where the summation is over the same $\nu$ as in (2.3), and

$$
\eta_{n}>\max \left\{\frac{2}{X_{n+1}-X_{n}}, 4 L \frac{X_{n+1}-X_{n}}{X_{n+1}}, 8 \frac{\epsilon\left(X_{n}\right) X_{n+1}}{X_{n+1}-X_{n}}\right\} \text {. }
$$

The sequence $\left\{\lambda_{n}\right\}$ is now modified as follows. If the number of $\lambda$ 's in $\left(X_{n}, X_{n+1}\right)$ is not greater than

$$
\left(L+\eta_{n}\right)\left(X_{n+1}-X_{n}\right)
$$

all the $\lambda$ in this interval are retained (" unmodified interval "). If the number of $\lambda$ 's in $\left(X_{n}, X_{n+1}\right)$ is greater than (2.7), then $\lambda$ 's from this interval are dropped out until the number of remaining $\lambda$ is at most equal to (2.7). For the modified sequence obviously

$$
\varlimsup \frac{l(r)}{r}=\varlimsup \frac{\Lambda\left(X_{n}\right)}{X_{n}}-L \leqslant 0 .
$$

Further, in a "modified interval" for the new sequence

$$
\begin{aligned}
\phi\left(X_{n+1}\right)-\phi\left(X_{n}\right) & =\underset{X_{n}<\lambda<X_{n+1}}{\sum} \frac{1}{\lambda}-L \log \frac{X_{n+1}}{X_{n}} \\
& >\frac{\left(L+\eta_{n}\right)\left(X_{n+1}-X_{n}\right)-1}{X_{n+1}}+L \log \left(1-\frac{X_{n+1}-X_{n}}{X_{n+1}}\right) .
\end{aligned}
$$

For sufficiently small positive $\xi$

$$
\log (1-\xi)>-\xi-\xi^{2}
$$

Hence, for sufficiently large $n$,

By (2.6),

$$
\phi\left(X_{n+1}\right)-\phi\left(X_{n}\right)>\frac{\eta_{n}\left(X_{n+1}-X_{n}\right)}{X_{n+1}}-\frac{1}{X_{n+1}}-L\left(\frac{X_{n+1}-X_{n}}{X_{n+1}}\right)^{2} .
$$

$$
1<\frac{1}{2} \eta_{n}\left(X_{n+1}-X_{n}\right) ; \quad L \frac{X_{n+1}-X_{n}}{X_{n+1}}<\frac{1}{4} \eta_{n} ; \quad \epsilon\left(X_{n}\right)<\frac{1}{8} \eta_{n} \frac{X_{n+1}-X_{n}}{X_{n+1}} .
$$


Hence

$$
\phi\left(X_{n+1}\right)-\phi\left(X_{n}\right)>\frac{1}{4} \eta_{n} \frac{X_{n+1}-X_{n}}{X_{n+1}}>\frac{1}{8} \eta_{n} \frac{X_{n+1}-X_{n}}{X_{n+1}}+\epsilon\left(X_{n}\right) .
$$

Therefore, for the new sequence,

$$
\begin{aligned}
\phi\left(X_{q}\right)-\phi\left(X_{p}\right) & =\sum_{k=p}^{q-1}\left[\phi\left(X_{k+1}\right)-\phi\left(X_{k}\right)\right] \\
& >-\epsilon\left(X_{p}\right)+\frac{1}{8} \Sigma^{\prime} \eta_{k} \frac{X_{k+1}-X_{k}}{X_{k+1}},
\end{aligned}
$$

where the summation is extended over the "modified intervals". This is an immediate consequence of (2.8) if every set of consecutive unmodified intervals is grouped with the preceding modified interval (if any). (1.9) follows at once from (2.9), and (1.8) is also a consequence of $(2.9)$ because of $(2.5)$.

3. In this section I prove that the conclusion of Theorem B is false if there are large intervals in which the density of the $\lambda$ 's falls appreciably below the value $1 / L$. More precisely, we make the following

Assumption A. There are constants $M, \beta, \gamma, M<L, 0<\beta<\gamma$ and a sequence of $X \rightarrow \infty$ such that for $u$ in the "exceptional interval"

$J$ :

$$
\begin{gathered}
X \leqslant u \leqslant Y, \quad(1+\beta) X \leqslant Y<(1+\gamma) X, \\
\Lambda(u)-\Lambda(X) \leqslant M(u-X) .
\end{gathered}
$$

Or, in other words,

$$
l(u)-l(X) \leqslant-(L-M)(u-X) .
$$

Then for $Z=(1+\beta) X$

$$
\begin{aligned}
\phi(Z)-\phi(X) & =Z^{-1} l(Z)-X^{-1} l(X)+\int_{X}^{Z} u^{-2} l(u) d u \\
& =Z^{-1}\{l(Z)-l(X)\}+\int_{X}^{Z} u^{-2}\{l(u)-l(X)\} d u \\
& <Z^{-1}\{l(Z)-l(X)\}<-(L-M) \beta(1+\beta)^{-1},
\end{aligned}
$$

so that (1.9) is not satisfied under Assumption A.

Before constructing the required counter-example I prove that one may assume without loss of generality that in the interval (3.1) also

$$
\Lambda_{0}(Y)-\Lambda_{0}(u) \leqslant M(Y-u),
$$

where $\Lambda_{0}(u)$ is the number of $\lambda^{\prime}$ s less than $u$. 
Choose $N, M<N<L$. Let $Z$ be the least upper bound of all $W \leqslant(1+\beta) X$ such that

$$
\Lambda_{0}(W)-\Lambda_{0}(u) \leqslant N(W-u) \quad(X \leqslant u \leqslant W) .
$$

If $Z=(1+\beta) X$, we need only replace $M$ by $N$ and $Y$ by $(1+\beta) X$ and the assertion is proved. If $Z<(1+\beta) X$, then for $Z<v \leqslant(1+\beta) X$ there is a $u=u(v) \geqslant X$ such that

$$
\Lambda_{0}(v)-\Lambda_{0}(u)>N(v-u)
$$

We can assume $u \geqslant Z$, since otherwise $u$ could be replaced by $Z$ :

$$
\begin{aligned}
\Lambda_{0}(v)-\Lambda_{0}(Z) & =\Lambda_{0}(v)-\Lambda_{0}(u)-\left[\Lambda_{0}(Z)-\Lambda_{0}(u)\right] \\
& >N(v-u)-N(Z-u)=N(v-Z) .
\end{aligned}
$$

In particular there will be a sequence of $Y$ 's $\geqslant Z$,

with

$$
(1+\beta) X=Y_{0}>Y_{1}>Y_{2} \ldots,
$$

$$
\begin{gathered}
N\left(Y_{0}-Y_{1}\right)<\Lambda_{0}\left(Y_{0}\right)-\Lambda_{0}\left(Y_{1}\right), \\
N\left(Y_{1}-Y_{2}\right)<\Lambda_{v}\left(Y_{1}\right)-\Lambda_{0}\left(Y_{2}\right), \text { etc. }
\end{gathered}
$$

This chain of inequalities breaks off when finally $Y_{k}=Z$. This index $k$ exists, since every interval $\left(Y_{h}, Y_{h-1}\right)$ contains at least one $\lambda$ and the number of $\lambda$ in $\left(Z, Y_{0}\right)$ is finite. Addition of the inequalities gives

$$
\begin{aligned}
N[(1+\beta) X-Z] & <\Lambda_{0}[(1+\beta) X]-\Lambda_{0}(Z) \\
& \leqslant \Lambda_{0}[(1+\beta) X]-\Lambda_{0}(X) \\
& \leqslant \Lambda[(1+\beta) X]-\Lambda(X)+1 \\
& \leqslant M \beta X+1
\end{aligned}
$$

by (3.2). Hence

$$
\begin{aligned}
Z & \geqslant\left[(1+\beta)-\frac{M}{N} \beta\right] X-\frac{1}{N} \\
& \geqslant\left(1+\beta^{\prime}\right) X
\end{aligned}
$$

for some positive $\beta^{\prime}=\beta^{\prime}(\beta, M, N)$ and sufficiently large $\boldsymbol{X}$. This shows that we may assume (3 . 3), changing $M$ into $N$ and $\beta$ into $\beta^{\prime}$ in Assumption A, if necessary. 
One aim is to show that under Assumption A there is a function $f(z)$ satisfying (1.2), but not (1.1). Let

$$
g(z)=\pi^{-1}(L z)^{1-2 L z} \sin \pi L z \Gamma^{2}(L z) e^{20 L e},
$$

where $C$ is Euler's constant. Then

$$
\begin{aligned}
g(z) & =(L z)^{-2 L z} \prod_{n=1}^{\infty} \frac{\mu_{n}-z}{\mu_{n}+z} e^{2 z / \mu_{n}}, \\
\mu_{n} & =n / L .
\end{aligned}
$$

We shall assume that there is an absolute constant $\delta>0$ such that all the end points of "exceptional intervals" (3.1) are at a distance greater than $\delta$ from the $\mu_{n}$. This can be brought about, if necessary, by shortening the exceptional intervals slightly (with a consequent small alteration in the value of $M$ ).

To construct $f(z)$ a sub-sequence $\left\{J_{v}\right\}$ of the intervals (3.1) is chosen for which $X_{v+1} / X_{v} \rightarrow \infty$ very rapidly. The counter-example is then provided by

$$
f(z)=g(z) \prod_{\nu} P_{\nu}(z)
$$

where

$$
P_{\nu}(z)=\prod_{\mu \in J_{\nu}} \frac{\mu+z}{\mu-z} e^{-2 z / \mu} \prod_{\lambda \in J_{\nu}} \frac{\lambda-z}{\lambda+z} e^{2 z / \lambda}\left(\frac{a_{\nu}-z}{a_{\nu}+z}\right)^{q} e^{2 z q_{\nu} / a_{\nu}}
$$

and $a_{v}$ and the positive integer $q_{v}$ are chosen so that

$$
-\sum_{\mu \in J_{\nu}} \mu^{-1}+\sum_{\lambda \in J_{\nu}} \lambda^{-1}+q_{\nu} / a_{\nu}=0 .
$$

To prove that $f(z)$ satisfies (1.2) but not (1.1) we need first some facts about the behaviour of $P_{v}(z)$. To save writing, the index $\nu$ will be omitted as long as we deal with one particular $J_{v}=J$. By (3.5)

$$
P(z)=\prod_{\mu \varepsilon J} \frac{\mu+z}{\mu-z} \prod_{\lambda \varepsilon J} \frac{\lambda-z}{\lambda+z}\left(\frac{a-z}{a+z}\right)^{q} .
$$

LEMMa 1. Let $Q(z)=z^{-1} \log |P(z)|$ where $P(z)$ is given by (3.6). Then, for given $\eta, 0<\eta<1$, the integer $q$ and the number $a$ in (3.5) can be chosen so that

$$
\frac{1}{2} \eta X<a<\eta X
$$

provided that $\boldsymbol{X}$ is sufficiently large. Also, if $\eta$ in (3.7) is small,

$$
\begin{array}{rlrl}
Q(z) & =O\left((|z| \mid a)^{2}\right)=O\left((|z| \mid X)^{2}\right) & & \left(|z|<\frac{1}{2} a\right), \\
& =O\left((Y /|z|)^{2}\right) & (|z|>2 Y),
\end{array}
$$


and

$$
\begin{array}{cl}
0<Q(x)<Q(Y) & (x>Y) \\
Q(x)<Q(X) & (a<x<X), \\
Q(x)<0 & (x<a) .
\end{array}
$$

Proof. By (3.2) and (3.3) the largest possible value of $\Sigma \lambda^{m}$ is attained if the $\lambda$ are evenly spaced at distances $1 / M$. Hence

$$
\sum_{J} \lambda^{-1} \leqslant M \log (Y / X)
$$

while

$$
\sum_{J} \mu^{-1} \approx L \log (Y / X)
$$

For integers $m$ other than -1 ,

$$
\underset{J}{\sum} \lambda^{m}<\sum_{J} \mu^{m}<A\left(Y^{1-m}+X^{1-m}\right),
$$

where $A$ is an absolute constant. Use of these estimates in (3.5) shows that $a>0$ so that, by suitable choice of the positive integer $q,(3.7)$ can be satisfied.

$$
\begin{aligned}
\operatorname{In}|z| & <a, \\
& z^{-1} \log P(z)=2 \sum_{n=1}^{\infty} \frac{z^{2 n}}{2 n+1}\left\{\sum_{J} \mu^{-2 n-1}-\sum_{J} \lambda^{-2 n-1}-q a^{-2 n-1}\right\},
\end{aligned}
$$

and the estimates for the sums given above show that the coefficient of $z^{2 n}$ is $O\left(a^{2 n}\right)$ and that it is negative for $\eta<\eta_{0}(M, L, \beta, \gamma)$. This proves the statements about $Q$ in the range $|z|<a$. The other statements are proved similarly.

I return to the function $f(z)$. It is not hard to see that it satisfies (1.2) (see Lemma 3 of [3] for a closely related calculation). I show now that $f(z)$ does not satisfy $(1,1)$ if the $X_{v}$ increases very rapidly.

If $E$ is the set of all $x$ not belonging to the $J_{v}$, then

$$
\varlimsup \lambda_{n}^{-1} \log \left|f\left(\lambda_{n}\right)\right| \leqslant \varlimsup_{\substack{x \rightarrow \infty \\ x \in E}} x^{-1} \log |f(x)|,
$$

since $f\left(\lambda_{n}\right)=0$ for $\lambda_{n} \varepsilon J$. It is therefore sufficient to prove that

$$
\varlimsup_{\substack{x \rightarrow \infty \\ x \in \mathbb{E}}} x^{-1} \log |f(x)|<\varlimsup_{x \rightarrow \infty} x^{-1} \log |f(x)| .
$$

By. Stirling's formula,

$$
x_{n}^{-1} \log \left|g\left(x_{n}\right)\right| \rightarrow 2 L(C-1)=\varlimsup_{x \rightarrow \infty} x^{-1} \log |g(x)|
$$


as $x_{n} \rightarrow \infty$ through any set of values whose distance from the set of points $\mu_{n}=n / L(n=1,2, \ldots)$ is positive.

If the $X_{\nu}$ are rapidly increasing, by (3.8) and (3.9)

$$
x^{-1} \log \left|\Pi P_{\bullet}(x)\right|=x^{-1} \log \left|P_{k}(x)\right|+o(1),
$$

as $x \rightarrow \infty$, where $J_{k}$ is the interval closest to $x$. (3.13) is therefore a consequence of the following statement.

There is a constant $\alpha>0$ such that every $J$ contains a point $\xi$ at a distance $\delta>0$ from every $\mu_{n}$ such that

$$
\xi^{-1} \log |P(\xi)|=Q(\xi)>Q(x)+\alpha \quad(x \notin J) .
$$

By $(3.10),(3.11)$ and $(3.12)$ it is enough to prove that $\xi$ can be chosen so that

$$
Q(\xi)>\max (Q(X), Q(Y))+\alpha .
$$

This will follow if we can find a $\xi$ such that

$$
Q(\xi)>Q(X)+\alpha
$$

and a $\xi$ (which might be different) such that

$$
Q(\xi)>Q(Y)+\alpha \text {. }
$$

I shall only show how to satisfy (3.14). The treatment of (3.15) is analogous.

Consider

$$
\Delta=Q(\xi)-Q(X) \quad(\xi \varepsilon J) .
$$

The contribution of a particular $\lambda$ to $\Delta$ is

$$
D(\lambda)=\xi^{-1} \log \left|\frac{\lambda-\xi}{\lambda+\xi}\right|-X^{-1} \log \left|\frac{\lambda-X}{\lambda+\bar{X}}\right| .
$$

Differentiation with respect to $\lambda$ shows that $D(\lambda)$ decreases if a $\lambda>X$ is moved closer to $\xi$. Also $D(\lambda) \rightarrow 0$ as $\lambda \rightarrow \infty$, and so $D(\lambda)<0$ for $\lambda>\xi$. Hence $\Delta$ is decreased if the $\lambda$ inside $J$ are moved closer to $\xi$ and if further $\lambda$ are added between $\xi$ and $Y$ ( $a$ and $q$ being kept fixed).

Next we choose a suitable $\xi$ with the aid of

Cartan's Lemma. Given $H>0$ and $p$ points $\lambda_{1}, \ldots, \lambda_{p}$ in the complex plane, we can find a set of at most $p$ circles with sum of radii less than $2 H$ such that for every $\xi$ outside these circles

$$
\left|\xi-\lambda_{n}\right|>m H / p \quad(n=1,2, \ldots, p)
$$


with the exception of, at most, $m-1$ of the $\lambda$ 's. (For a proof of the Lemma in this form see [6] with $\alpha=1$.)

We apply the Lemma to the $\lambda$ 's between $X$ and $(1+k) X$, where $0<k<\beta$. The number, $p$, of such $\lambda$ 's is at most $k M X$, by (3.2). We choose $H=k X / 8$. The circles of Cartan's Lemma cover at most a set of measure $4 H=k X / 2$ on the real axis. There is therefore outside the Cartan circles a

with

$$
\begin{gathered}
\xi=(1+\sigma) X \\
\frac{1}{5} k<\sigma<\frac{4}{5} k, \\
\left|\xi-\mu_{n}\right|>\delta \quad(n=1,2, \ldots) .
\end{gathered}
$$

For this $\xi$ there is

$$
\text { no } \lambda \text { satisfying }|\xi-\lambda|<(k X / 8) / k M X=1 / 8 M,
$$

at most one $\lambda$ satisfying $|\xi-\lambda|<2 / 8 M$,

at most two $\lambda$ satisfying $|\xi-\lambda|<3 / 8 M$,

We shall now estimate $\Delta$ under the assumption that there is

$$
\begin{aligned}
& \text { one } \lambda \text { at a distance } 1 / 8 M \text { from } \xi \text {, } \\
& \text { one } \lambda \text { at a distance } 2 / 8 M \text { from } \xi \text {, } \\
& \text { one } \lambda \text { at a distance } s / 8 M \text { from } \xi \text {, }
\end{aligned}
$$

where $s(\geqslant p)$ is the largest integer not exceeding $k M X$, while the remaining $\lambda$ are equally spaced between $(1+k) X$ and $Y$ with a distance $1 / M$ between nearest neighbours. This position of the $\lambda$ can be obtained from their original distribution by moving the $\lambda$ closer to $\xi$ and, perhaps, adding further $\lambda>\xi$. Both these operations decrease $\Delta$, so that it will be sufficient to prove $(3.14)$ in this special case. Now we have

$$
|R(\xi)|=\left|\prod_{\lambda \in J} \frac{\lambda-\xi}{\lambda+\xi}\right| \geqslant \prod_{n=1}^{\varepsilon} \frac{n / 8 M}{\xi+(n / 8 M)+\xi} \prod_{m=m_{1}}^{m_{\mathrm{z}}} \frac{m / M-\xi}{m / M+\xi},
$$

where $m_{1}$ and $m_{2}$ are such that $m$ ranges over all integers satistying

$$
(1+k) X<m / M<Y \text {. }
$$

Or, in terms of $\Gamma$-functions,

$$
|R(\xi)| \geqslant \frac{\Gamma(s+1) \Gamma(16 M \xi)}{\Gamma(16 M \xi+s+1)} \frac{\Gamma\left(m_{2}-M \xi+1\right) \Gamma\left(m_{1}+M \xi\right)}{\Gamma\left(m_{1}-M \xi\right) \Gamma\left(m_{2}+M \xi+1\right)} .
$$


The further calculation is simplified if it is remarked that a change of $O(1)$ in the argument of any one of the $\Gamma$-functions changes the logarithm of this $\Gamma$-function by $O(\log X)$. This enables us to write $(\xi=(1+\sigma) X)$

$$
\begin{aligned}
\log |R(\xi)| & \geqslant \log \Gamma(k M X)+\log \Gamma(16(1+\sigma) M X) \\
& -\log \Gamma(16(1+\sigma) M X+k M X) \\
& +\log \Gamma(M Y-(1+\sigma) M X) \\
& +\log \Gamma((1+k) M X+(1+\sigma) M X) \\
& -\log \Gamma((1+k) M X-(1+\sigma) M X) \\
& -\log \Gamma(M Y+(1+\sigma) M X) \\
& +O(\log X) .
\end{aligned}
$$

Or, by Stirling's Formula,

Similarly

$$
\begin{aligned}
\log |R(\xi)| & \geqslant k M X \log k+16(1+\sigma) M X \log 16(1+\sigma) \\
& -\{16(1+\sigma)+k\} M X \log \{16(1+\sigma)+k\} \\
& +\{M Y-(1+\sigma) M X\} \log \{(Y / X)-(1+\sigma)\} \\
& +(2+k+\sigma) M X \log (2+k+\sigma) \\
& -\{M Y+(1+\sigma) M X\} \log \{(Y / X)+1+\sigma\} \\
& +O(\log X) .
\end{aligned}
$$

$$
\begin{aligned}
\log |R(X)| & \leqslant(8 \sigma+k) M X \log (8 \sigma+k) \\
& -8 \sigma M X \log 8 \sigma \\
& +(16+8 \sigma) M X \log (16+8 \sigma) \\
& -(16+8 \sigma+k) M X \log (16+8 \sigma+k) \\
& +(M Y-M X) \log \{(Y / X)-1\} \\
& -k M X \log k \\
& +(2+k) M X \log (2+k) \\
& -(M Y+M X) \log \{(Y / X)+1\} \\
& +O(\log X)
\end{aligned}
$$


Now we choose $k$ rather small and remember (3.16). Collecting those terms in which the logarithm is equal to $\log k+O(1)$ and those in which the logarithm is $O(1)$, we obtain finally

$$
\xi^{-1} \log |R(\xi)|-X^{-1} \log |R(X)| \geqslant M k \log k+O\left(k+\frac{\log X}{X}\right)
$$

where the constant implied in the $O$-notation depends on $M$ only.

Similarly, 1 if

$$
S(x)=\prod_{\mu_{n} \varepsilon J} \frac{\mu_{n}+x}{\mu_{n}-x}
$$

then

$$
\xi^{-1} \log |S(\xi)|-X^{-1} \log |S(X)|>-L k \log k+O\left(k+\frac{\log X}{X}\right) .
$$

Finally, if

$$
T(x)=\left(\frac{a-x}{a+x}\right)^{q}
$$

then

$$
\begin{aligned}
\xi^{-1} \log |T(\xi)|- & X^{-1} \log |T(X)| \\
& =q X^{-1}\left\{(1+\sigma)^{-1} \log \frac{1+\sigma-a / X}{1+\sigma+a / X}-\log \frac{1-a / X}{1+a / X}\right\} \\
& =O(\sigma)=O(k),
\end{aligned}
$$

by $(3.5)$ and $(3.10)$.

Now (3.14) is a consequence of $(3.6),(3.17),(3.18)$ and $(3.19)$.

4. To prove that (1.9) is a necessary condition for the assertion of Theorem B we must still consider the case when both (1.9) and Assumption A of the last section do not hold.

If Assumption $A$ is false, then for every $\epsilon, 0<\epsilon<1$, we can find a $U=U(\epsilon)$ such that for every $u>U$ there is a $v$ satisfying

$$
\Lambda(v)-\Lambda(u)>L(1-\epsilon)(v-u) \quad(u<v<(1+\epsilon) u) .
$$

Since the interval $(u, v)$ must contain at least one $\lambda$, every interval $(X, Z)$ with $X>U$ can be covered by a finite number of non-overlapping

\footnotetext{
1 Here we use the fact that both $\xi$ and $X$ are at a distence greater than $\delta$ from the neareet $\mu_{n}$.
} 
intervals $(u, v)$. Therefore

$$
\begin{aligned}
\phi(Z)-\phi(X) & =\sum_{X<\lambda<Z} \lambda^{-1}-L \log (Z / X) \\
& >\Sigma \frac{L(1-\epsilon)(v-u)}{v}-L \log (Z / X) \\
& \geqslant L \frac{1-\epsilon}{1+\epsilon} \Sigma \frac{v-u}{u}-L \log (Z / X) \\
& >L \frac{1-\epsilon}{1+\epsilon} \int_{X}^{Z} \frac{d t}{t}-L \log (Z / X) \\
& >-\frac{2 \epsilon L}{1+\epsilon} \log (Z / X) .
\end{aligned}
$$

On the otber hand, since (1.9) is false, there is an $a>0$ and an infinite sequence of intervals $J_{v}=\left(X_{v}, Y_{v}\right) \quad\left(X_{v} \rightarrow \infty\right)$ such that

$$
\phi\left(Y_{v}\right)-\phi\left(X_{v}\right)=-a \text {. }
$$

This is compatible with (4.2) only if

$$
Y_{v} / X_{v} \rightarrow \infty
$$

We may also suppose that on an exceptional interval $(X, Y)$

$$
-a \leqslant \phi(Z)-\phi(X)<0 \quad(X<Z<Y)
$$

(otherwise replace $X$ by a larger, $Y$ by a smaller number). Therefore, if $X_{v}<Z<Z^{\prime}<Y_{v}$,

$$
\phi\left(Z^{\prime}\right)-\phi(Z)<a,
$$

and reasoning similar to that leading to $(4.2)$ proves

Lemma 2. For given positive numbers $R, \epsilon, R>\epsilon$, and $L^{\prime}>L$ there is a constant $K=K\left(L^{\prime}, R, \epsilon\right)$ such that for all large $\nu$ the interval $\left(X_{v}, K X_{v}\right)$ contains an interval $\left(Z, Z^{\prime}\right)$ with $Z^{\prime} \mid Z=R$ such that for $Z \leqslant u,(1+\epsilon) u \leqslant Z^{\prime}$

$$
\Lambda((1+\epsilon) u)-\Lambda(u)<L^{\prime} \epsilon u .
$$

[If this is not true for an interval $(Z, R Z)$, then ultimately (for large $v$ ) $\phi(R Z)-\phi(Z)>k\left(L^{\prime}, \epsilon\right)>0$. There cannot be too many consecutive such intervals, by (4.5).] 
Next we define a function $f(z)$ depending on a parameter $\epsilon>0$ which will provide a counter-example for sufficiently small $\epsilon$.

We select a sub-sequence $\left\{J_{v}\right\}$ of the exceptional intervals such that

$$
\begin{aligned}
Y_{\nu} & >X_{\nu} \epsilon^{-2}, \\
X_{\nu+1} & >Y_{\nu} \epsilon^{-2} .
\end{aligned}
$$

In view of (4.3) this is always possible. The sequence $\left\{k_{n}\right\}$ is defined as follows. Outside the $J_{v}$ the $k$ are evenly spaced at distances $1 / L$, inside a $J_{v}$ every $\lambda$ belongs to $\left\{\dot{k}_{n}\right\}$ and in addition further $k$ 's are added between $X_{v}$ and $X_{v} / 2 \epsilon$ such that the minimum distance of two $k$ 's is greater than $\frac{1}{3} c[c$ of $(1.3)]$ and

$$
\sum_{\boldsymbol{k}: \mathrm{J}_{\nu}} k^{-1}-L \log \left(Y_{\nu} / X_{\nu}\right)=0
$$

These requirements are compatible if $\epsilon$ is sufficiently small. Note that there is a constant $b$ such that

$$
\sum_{k<x} k^{-1}-L \log x \rightarrow b
$$

as $x \rightarrow \infty$ through any sequence outside the $J_{v}$.

Put

$$
f(z)=z^{-2 L z} \prod_{n=1}^{\infty} \frac{k_{n}-z}{k_{n}+z} e^{2 z / k_{n}} .
$$

It is easily seen that $f(z)$ satisfies (1.2).

I prove next that for $x$ outside the $J_{v}$

$$
\varlimsup x^{-1} \log |f(x)|<2 b-2 L+\rho,
$$

where $\rho$ here and everywhere in this section denotes a number which can be made arbitrarily small by choosing $\epsilon$ sufficiently small. We shall need the following, easily proved, estimates of

$$
\begin{array}{cl}
W=\log \left|\frac{k-x}{k+x}\right|+\frac{2 x}{k} \\
W<0 & (0<x<k) \\
W=O\left(x^{3} / k^{3}\right) & \left(0<x / k<\frac{1}{2}\right) \\
W<2 x / k & (0<x / k) \\
W=2 x / k+O(k / x) & \left(0<k / x<\frac{1}{2}\right) .
\end{array}
$$


Let $x$ be outside the $J_{v}$,

$$
\begin{aligned}
\log |f(x)| & =-2 L x \log x+\sum_{k \in e x}+\sum_{e x<k<x / e}+\sum_{k \geqslant x / e}\left\{\log \left|\frac{k-x}{k+x}\right|+\frac{2 x}{k}\right\} \\
& =-2 L x \log x+\Sigma_{1}+\Sigma_{2}+\Sigma_{3} .
\end{aligned}
$$

By (4. 10)

$$
\Sigma_{1}<2 x \underset{k<e x}{\sum} k^{-1}
$$

By (4.8),

$$
\Sigma_{3}<0 \text {. }
$$

Also, by (4.8), $\Sigma_{2}$ is increased if any terms arising from $k$ 's inside a $J_{v}$ which are not $\lambda$ 's are omitted, for such $k$ are necessarily greater than $x$. This changes $\Sigma_{2}$ to $\Sigma_{2}{ }^{\prime}$, say. All $k$ contributing to $\Sigma_{2}{ }^{\prime}$ are either outside the $J_{v}$ or belong to $\{\lambda\}$. It follows that the range of summation can be covered by a finite number of non-overlapping intervals $(u, v)$ for which (4.1) holds, where $\Lambda(t)$ is the number of $k$ 's not greater than $t$ contributing to $\Sigma_{2}{ }^{i}$. Hence

$$
\begin{aligned}
\Sigma_{2}^{\prime} \log \left|\frac{k-x}{k+x}\right| \leqslant L(1-\epsilon) \sum_{v<x}(v-u) \log \frac{x-u}{x+u}+L(1-\epsilon) \sum_{u>x}(v-u) \log \frac{v-x}{v+x} \\
\leqslant L(1-\epsilon) x \sum_{v<x}\left(\frac{v}{x}-\frac{u}{x}\right) \log \frac{1-u / x}{1+u / x} \\
+L(1-\epsilon) x \sum_{u>x}\left(\frac{v}{x}-\frac{u}{x}\right) \log \frac{v / x-1}{v / x+1} .
\end{aligned}
$$

The last two sums are Riemann sums approximating to

$$
\int_{e}^{1} \log \frac{1-u}{1+u} d u=-2 \log 2+O\left(\epsilon^{2}\right)
$$

and

$$
\int_{1}^{1 / \bullet} \log \frac{u-1}{u+1} d u=2 \log \epsilon-2+2 \log 2+O\left(\epsilon^{2}\right)
$$

respectively.

Further, because of the spacing of the $k$ and because of $(4.2)^{1}$ and $(4.5)$

$$
\Sigma_{2}^{\prime} \frac{2 x}{k}=\sum_{e x<k \leqslant x} \frac{2 x}{k}-2 L x \log \epsilon+o(x)
$$

1 The number $\epsilon$ in (4.2) is not the same $\epsilon$ as here. In $(4.2) \epsilon=o(1)$ as $X \rightarrow \infty$. 
as $x \rightarrow \infty$. Collecting the information on $\Sigma_{1}, \Sigma_{2}$ and $\Sigma_{3}$ and remembering (4.6) we obtain (4.7). In particular

$$
\varlimsup \varlimsup_{n}^{-1} \log \left|f\left(\lambda_{n}\right)\right| \leqslant 2 b-2 L+\rho,
$$

since $\log \left|f\left(\lambda_{n}\right)\right|=-\infty$ if $\lambda_{n} \in J_{v}$.

A contradiction to (1.1) will arise if there is a sequence $\left\{\xi_{v}\right\}$ such that $\xi, \varepsilon J$, and

$$
\varlimsup \lim \xi_{p}^{-1} \log \left|f\left(\xi_{\nu}\right)\right| \geqslant 2 b-2 L+2 a-\rho .
$$

By Lemma 2, with $R=\epsilon^{-4}, L^{\prime}=L(1+\epsilon)$, every interval $J_{*}\left(\nu>\nu_{0}\right)$ will contain an interval $I=\left(Z, Z \epsilon^{-4}\right)$ such that no interval $(u,(1+\epsilon) u) \subset I$ has more than $L(1+\epsilon) \epsilon u \lambda$ 's inside it. Also $Z / X$, is less than a constant depending on $\epsilon$ only. Let $\xi_{v}$ be as close to $Z \epsilon^{-2}$ as is compatible with the requirement that $\xi_{\nu}$ is at a distance $\geqslant \frac{1}{2} c$ from the nearest $k$. All $k$ 's in $\left(\epsilon \xi_{v}, \xi_{v} / \epsilon\right)$ are $\lambda$ 's, so that this last condition can be satisfied. To compute $\xi_{v}^{-1} \log \left|f\left(\xi_{v}\right)\right|$ we use the same notation as in $(4.12)$ with $\xi=\xi_{v}$ in place of $x$. By (4.9)

By (4.11),

$$
\Sigma_{3}>O\left(\xi^{3} \sum_{k<\xi / \epsilon} k^{-3}\right)=O\left(\xi^{3}(\xi / \epsilon)^{-2}\right)=O\left(\epsilon^{2} \xi\right)
$$

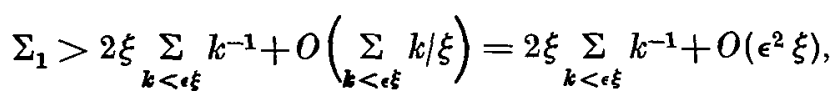

since the number of $k$ less than $\epsilon \xi$ is $O(\epsilon \xi)$.

All $k$ contributing to $\Sigma_{2}$ are members of $\{\lambda\}$. Using the upper bound for the number of $\lambda^{\prime} s$ in $(u,(1+\epsilon) u)$ found above and reasoning as before we have

$$
\sum_{e \xi<k<\xi /(1+a)}+\sum_{(1+e) \xi \leqslant k \leqslant \xi / \epsilon} \log \left|\frac{k-\xi}{k+\xi}\right|>(-2+2 \log \epsilon) L \xi-\rho \xi .
$$

Also, if $N$ is the number of $\lambda$ in $(\xi /(1+\epsilon),(1+\epsilon) \xi)$, then, by (1.3),

$$
\begin{aligned}
\sum_{\xi /(1+\bullet)<k<(1+\bullet) \xi} \log \left|\frac{k-\xi}{k+\xi}\right| & >\log \frac{\left(\frac{1}{2} c\right)^{2} \cdot\left(\frac{3}{2} c\right)^{2} \cdot\left(\frac{5}{2} c\right)^{2} \ldots\left(\frac{N-1}{2} c\right)^{2}}{[(2+\epsilon) \xi]^{N}}+O(\log \xi) \\
& >N \log N-N \log \xi+O(N) .
\end{aligned}
$$

But $N=O(\epsilon \xi)$ and so the right-hand side is

$$
O(\epsilon \xi \log 1 / \epsilon)>-\rho \xi
$$


As before

$$
\Sigma_{2} k^{-1}>\underset{\epsilon \xi k \leqslant \xi}{\sum} k^{-1}-L \log \epsilon-\rho
$$

Hence ultimately

$$
\xi^{-1} \log |f(\xi)|>2 \sum_{k \leqslant \xi} k^{-1}-2 L \log \xi-2 L-\rho .
$$

But if $\xi$ is in the exceptional interval $(X, Y)$,

$$
\begin{aligned}
\sum_{k<\xi} k^{-1}-L \log \xi=\left(\sum_{k<X} k^{-1}-L \log X\right) & +\left(\sum_{X<k<Y} k^{-1}-L \log (Y / X)\right) \\
& -\left(\sum_{\xi<k<Y} k-L \log Y / \xi\right) .
\end{aligned}
$$

As $X \rightarrow \infty$, the first term tends to $b$, the second is 0 and the last is

$$
-(\phi(Y)-\phi(\xi))=-(\phi(Y)-\phi(X))+(\phi(\xi)-\phi(X)) .
$$

By hypothesis the first part is $a$; by our choice of $\xi, \xi / X$ is less than some fixed number depending on $\epsilon$ only. Hence $\phi(\xi)-\phi(X) \rightarrow 0$. (4.13) now follows from (4.14). This completes the proof that (1.9) is a necessary condition.

5. It remains to prove the necessity of (1.8). Since we may assume (1.9), (1.8) can be replaced by

$$
\varlimsup_{r \rightarrow \infty} \phi(r)=\infty \text {. }
$$

But I have shown elsewhere [2, Theorem 1] that

$$
\varlimsup_{r \rightarrow \infty} \phi(r)<\infty
$$

implies the existence of a non-constant $f(z)$ satisfying (1.2) and such that $f\left(\lambda_{n}\right)=0$. For this $f(z)$ the left-hand side of $(1.1)$ is $-\infty$. But the right-hand side of (1.1) is greater than $-\infty$, by a Phragmén-Lindelöf theorem. Therefore the conclusion of Theorem B fails if (1.8) is not satisfied.

I am greatly indebted to the referee for many improvements of an earlier version of this paper. 


\section{REFERENCES.}

1. R. P. Boas, "The Growth of Analytic Functions ", Duke Math. Journal, 13 (1946), 471-481.

2. W. H. J. Fuchs, "A Generalization of Carleon's Theorem", Journal London Math. Soc., 21 (1946), 106-110.

3. W. H. J. Fuchs, "On the Closure of $\left\{e^{-t} \ell^{a_{\nu}}\right\} "$, Proc. Cambridge Phil. Soc., 42 (1046), 91-105.

4. N. Levinson, Gap and Density Theorems (New York, 1940).

5. A. J. Macintyre and W. H. J. Fuchs "Inequalities for Logarithmic Derivatives of a Polynomial ", Journal London Math. Soc., 15 (1940), 162-168.

Dlipartment of Mathematios,

CORNELL UNIVERSITY,

ITHACA, New York. 